\title{
6.2 FRAGMENTIERUNG ALS HERAUSFORDERUNG: EIN METHODOLOGISCHES RESÜMEE
}

So muss eine Wissenschaftssoziologie der Soziologie einerseits der Tatsache gerecht werden, dass die Forschenden in den eigenen Untersuchungskontext eingebunden sind. ${ }^{1}$ Das macht sich - im Falle einer qualitativen Untersuchung nicht nur durch besondere Anforderungen an die methodologische Umsetzung und die Erhebungsform bemerkbar, bspw. an die Interviewführung. Es stellt sich zudem die Frage, welche Methode die Perspektive auf das Wie eröffnen kann, um dem Anspruch der Offenheit der Analyse im Sinne eines sozialkonstruktivistischen Paradigmas der Grenzkonstruktionen gerecht zu werden. Die Auswahl fiel auf die dokumentarische Methode, die nach dem Was und dem Wie innerhalb hermeneutischer Verfahren fragt, ohne, wie die objektive Hermeneutik, von objektiven Sinnstrukturen auszugehen.

Die Frage, die sich aus methodologischer Sicht stellte, war, wie auf Basis diverser disziplinärer Charakteristika ein Sample für eine qualitative Untersuchung der Soziologie ausgewählt werden kann. Die Entwicklung eines solchen Samples stellt gleichzeitig den Beitrag einer Wissenschaftssoziologie der Soziologie dar. Die Kriterien wurden sowohl aus der Theorie abgeleitet als auch auf Basis empirischer Studien, wie dem Forschungsrating des Wissenschaftsrats. Hier galt es, Konvergenzen in der Disziplin zu finden, die für eine Robustheit der Auswahl der Untersuchungskriterien sprechen. Die Herausforderung, die sich bei der Auswahl eines qualitativen Samples stellt, ist die der großen internen Fragmentierung der Soziologie. Diese wurde bereits an anderer Stelle der Arbeit thematisiert. Sie ist auch damit verbunden, dass ein kognitiver Kern der Soziologie fehlt, was Wagner (1990) als mangelnde wissenschaftliche Konsolidierung bezeichnete. Zudem konstatieren Autoren wie Cole (1994) oder Stinchcombe (1994), dass die Soziologie aufgrund fluider Grenzen zu ihrem Untersuchungsgegenstand niemals einen solchen kognitiven Kern ausbilden wird. Die Antwort auf eine solche Herausforderung wurde deshalb erneut in der Zusammenführung zweier Zugänge, diesmal methodologischer Art, gesucht.

Hier wurde auf die Grounded Theory rekurriert, die mit ihrem Anspruch des „theoretischen Samplings“ und der Offenheit der Herangehensweise kompatibel

1 Wir erinnern uns an Breuer: „1. Die Standpunktgebundenheit des Wissens; 2. Die Kabinenhaftigkeit der Erkenntnis; 3. Die ,Sinnesgebundenheit', ,Konzept-/Schema-/ Sprachgebundenheit‘ und ,Instrumentengebundenheit' sowie 4. Die Interaktivität zwischen Erkenntnissubjekt und -Objekt bzw. das der Interventionshaftigkeit des epistemologischen Subjekt-Objekt-Kontaktes“. (Breuer, 2003, Sp. 4 ff.) 
mit der dokumentarischen Methode ist. Auf Basis einer ausführlichen Analyse ist es deshalb gelungen, ein Gerüst für eine empirische Analyse der Soziologie zu entwickeln, die sich als methodologischer Beitrag zu einer Wissenschaftssoziologie der Soziologie versteht. Da sich die Soziologie weder durch einen organisationalen noch aus einem thematischen Kontext heraus als stabil erweist, wurden Strukturierungsmomente gesucht, die für eine maximale und minimale Kontrastierung der Fälle geeignet erscheinen. Drei Strukturierungsmomente wurde für die Fallauswahl identifiziert: strukturelle Ähnlichkeit des Vergleichsfalls (z.B. der Disziplin), kognitive Affinität der jeweils zu untersuchenden Einheiten (Schulen bzw. Denktraditionen) und eine historische Regelmäßigkeit dessen, was untersucht werden soll, das bedeutet, dass sich die zu untersuchenden Debatten über einen historischen Zeitraum als stabil erweisen sollten (Verhältnis zum Untersuchungsgegenstand). Innerhalb dieser Strukturierungsmomente wurden wiederum in sich kontrastierende Denk- und Methodentraditionen ausgewählt, um die Fälle miteinander zu vergleichen. Dieses Modell ist auch auf andere qualitative Untersuchungen der Soziologie übertragbar.

Die Auswertung der Interviews hat gezeigt, dass sich die diversen Formen, wie die Interviewten epistemische Autorität und eine Situierung innerhalb der Community erzeugen, nicht eindeutig einer bestimmten Methoden- bzw. Theorietradition zuordnen lassen. Zudem wurde es bewusst vermieden, Kategorien im Sinne einer Burawoy'schen Systematik aufzustellen. Denn wären diese in Hinblick auf „,instrumentelles Wissen“ vs. „kritisches Wissen“ entstanden, so wäre das Zirkularitätsproblem in Hinblick auf die Empirie zur Falle geworden: Instrumentelles Wissen würde sich dann an einen quantitativen Forschungsstil anlehnen, der leicht mit einem positivistischen Paradigma in Verbindung gebracht werden kann, während kritisches Wissen einer Ausrichtung an der kritischen Theorie nahestehen würde. Dagegen wurde die Auswertung bewusst quer zu diesen Kategorien durchgeführt. Der Anspruch an jegliche Soziologie und umso mehr auch an eine Wissenschaftssoziologie der Soziologie ist deshalb, das theoretische Sampling und die Auswertung möglichst gegen den Strich zu bürsten, um altbekannte Kategorisierungen zu vermeiden und nicht, wie bereits in der Einleitung konstatiert, das bereits Bekannte in eine neue Ordnung zu bringen. 